\title{
Comparable plasma lipid changes in patients with high-grade cervical intraepithelial neoplasia and patients with cervical cancer
}

Miso Nam ${ }^{1, \dagger}$, Sang-Soo Seo ${ }^{2, \dagger}$, Sunhee Jung ${ }^{1,3}$, Seo Young Jang ${ }^{1,4}$, Jueun Lee ${ }^{1}$, Minji Kwon ${ }^{5}$, Imran Khan ${ }^{5}$, Do Hyun Ryu ${ }^{3}$, Mi Kyung Kim ${ }^{5, *}$, Geum-Sook Hwang ${ }^{1,4, *}$

${ }^{1}$ Integrated Metabolomics Research Group, Western Seoul Center, Korea Basic Science Institute, 150 Bugahyeon-ro, Seodaemun-gu, Seoul 03759, Korea

${ }^{2}$ Center for Uterine Cancer, National Cancer Center, Madu-dong, Ilsandong-gu, Goyang-si, Gyeonggi-do 10408, Korea

${ }^{3}$ Department of Chemistry, Sungkyunkwan University, Suwon 16419, Korea

${ }^{4}$ Department of Chemistry and Nano Science, Ewha Womans University, 52 Ewhayeodae-gil, Seodaemun-gu, Seoul 03760, Korea

${ }^{5}$ Division of Cancer Epidemiology and Prevention, National Cancer Center, Madu-dong, Ilsandong-gu, Goyang-si, Gyeonggi-do 10408, Korea

${ }^{6}$ Department of Food Science and Technology, The University of Haripur, Pakistan.

$\dagger$ These authors contributed equally to this work.

${ }^{* *}$ Corresponding author:

Mi Kyung Kim, PhD, National Cancer Center

E-mail: alrud@ncc.re.kr, Tel.: +82-31-920-2202, Fax: +82-31-920-2006

Geum-Sook Hwang, PhD, Korea Basic Science Institute

E-mail: gshwang@kbsi.re.kr, Tel.: +82-2-6908-6200, Fax: +82-2-6908-6239 


\section{Table of Content}

\section{Supporting figures}

Figure. S1 Representative total ion chromatogram of UPLC-QTOF-MS

Figure. S2 PCA score plots obtained from UPLC-QTOF-MS spectra of plasma lipids in positive and negative modes.

Figure. S3 PCA score plots obtained from the identified plasma lipids.

Figure. S4 Fold changes in the levels of lipids found in plasma samples from the CIN1 groups and cervical cancer groups and the CIN1 and CIN2/3 groups.

Figure. S5 A lipid correlation network was generated between the CIN1 groups and cervical cancer groups and between the CIN1 groups and CIN2/3 groups. 
A

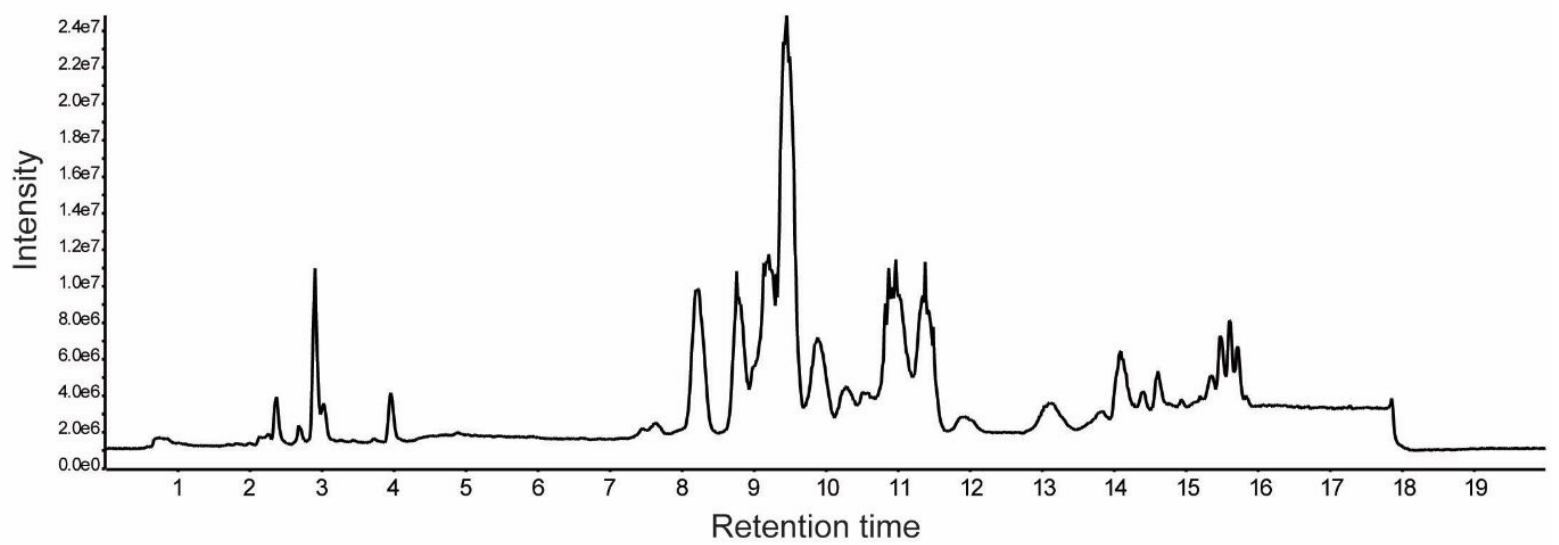

B

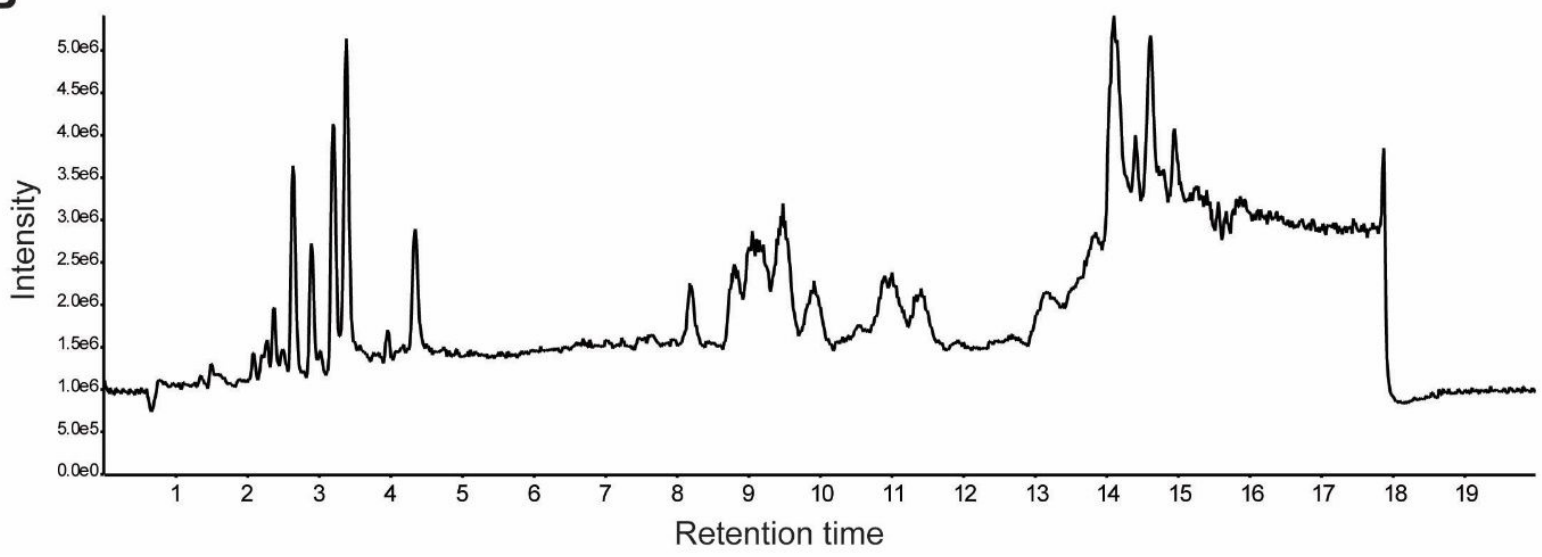

Figure S1. Representative total ion chromatogram of UPLC-QTOF-MS: (A) positive mode and (B) negative mode. 
A

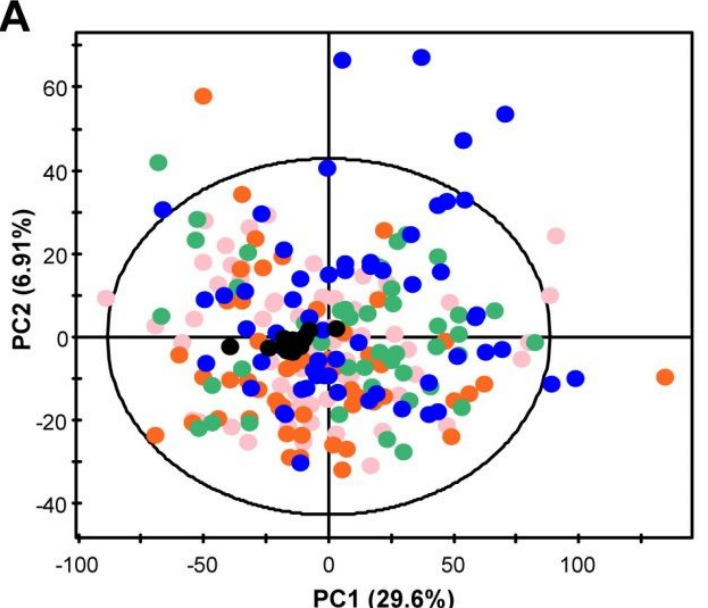

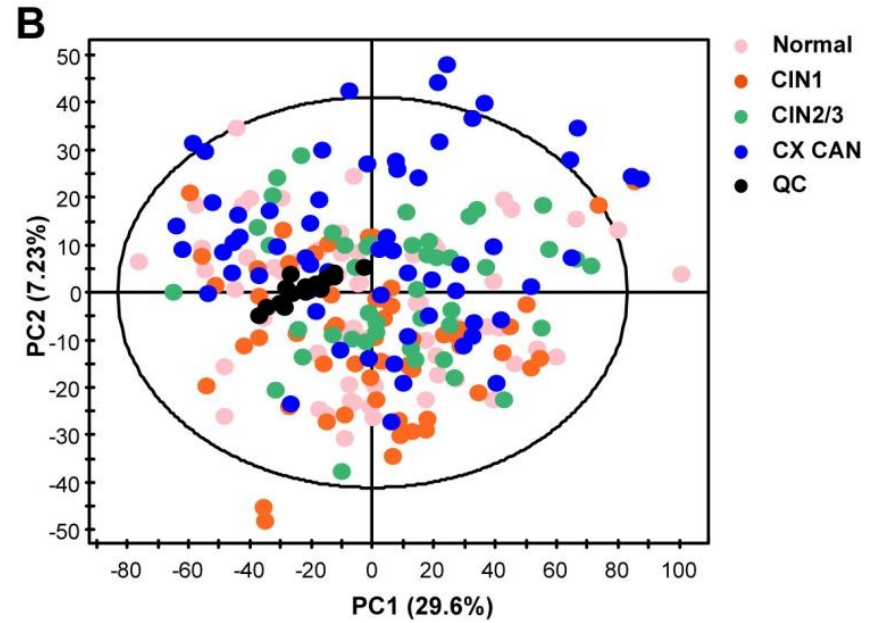

Figure S2. PCA score plots obtained from UPLC-QTOF-MS spectra of plasma lipids in (A) positive $\left(R^{2} X=0.744\right.$, and $\left.Q^{2}=0.594\right)$ and $(B)$ negative $\left(R^{2} X=0.725\right.$, and $\left.Q^{2}=0.6\right)$ modes. CIN1: cervical intraepithelial neoplasia 1, CIN2/3: cervical intraepithelial neoplasia 2 or 3, CX CAN: cervical cancer, QC: quality control. 
A

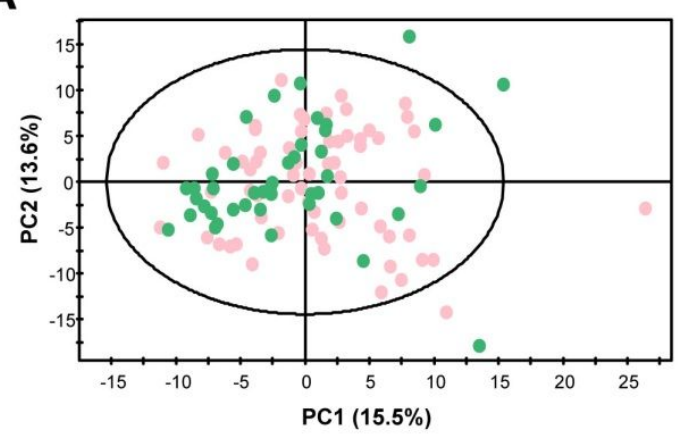

C

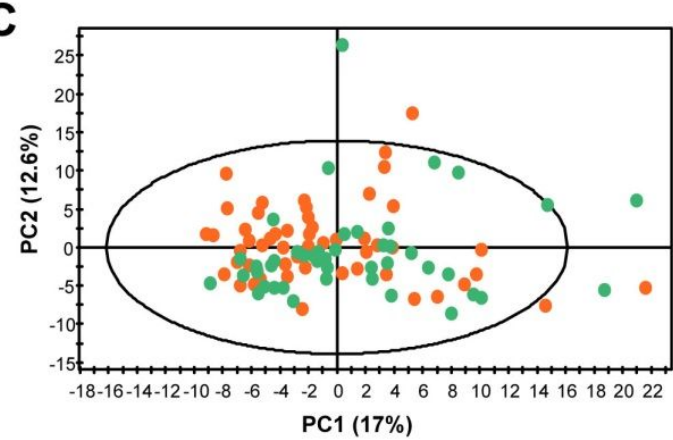

B

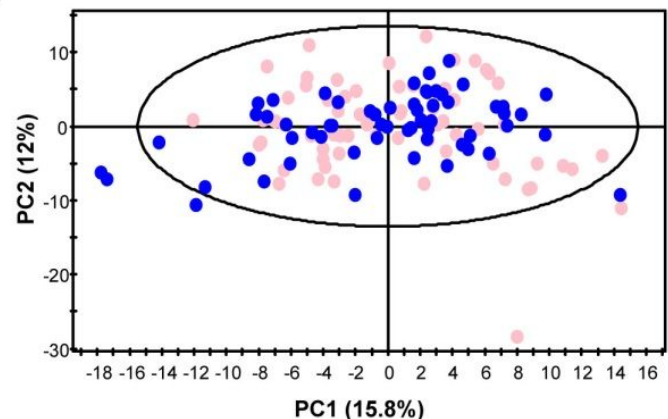

Normal - CIN 1 - CX CAN

D

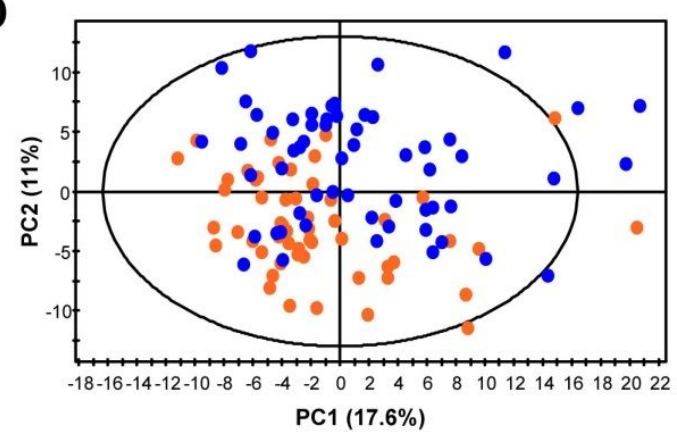

Figure S3. PCA score plots obtained from the identified plasma lipids. Comparisons were made between normal controls and $\mathrm{CIN} 2 / 3$ patients $\left(\mathrm{A}: \mathrm{R}^{2} \mathrm{X}=0.788\right.$ and $\left.\mathrm{Q}^{2}=0.596\right)$, normal controls and CX CAN patients (B: $\mathrm{R}^{2} \mathrm{X}=0.802$ and $\left.\mathrm{Q}^{2}=0.541\right), \mathrm{CIN} 1$ and $\mathrm{CIN} 2 / 3$ patients $(\mathrm{C}$ : $\mathrm{R}^{2} \mathrm{X}=0.782$ and $\left.\mathrm{Q}^{2}=0.513\right)$, and $\mathrm{CIN} 1$ and $\mathrm{CX}$ CAN patients $\left(\mathrm{D}: \mathrm{R}^{2} \mathrm{X}=787\right.$ and $\left.\mathrm{Q}^{2}=0.519\right)$. CIN1: cervical intraepithelial neoplasia 1, CIN2/3: cervical intraepithelial neoplasia 2 or 3, CX CAN: cervical cancer. 


\section{a}

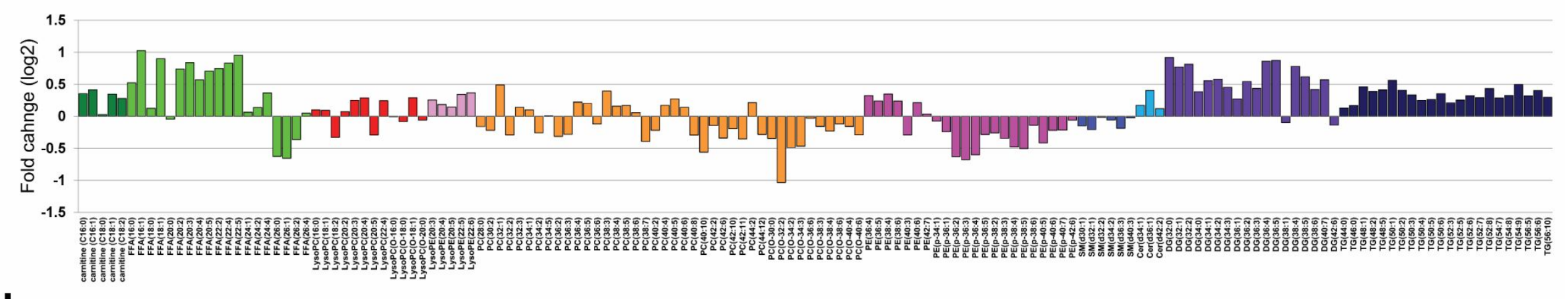

b

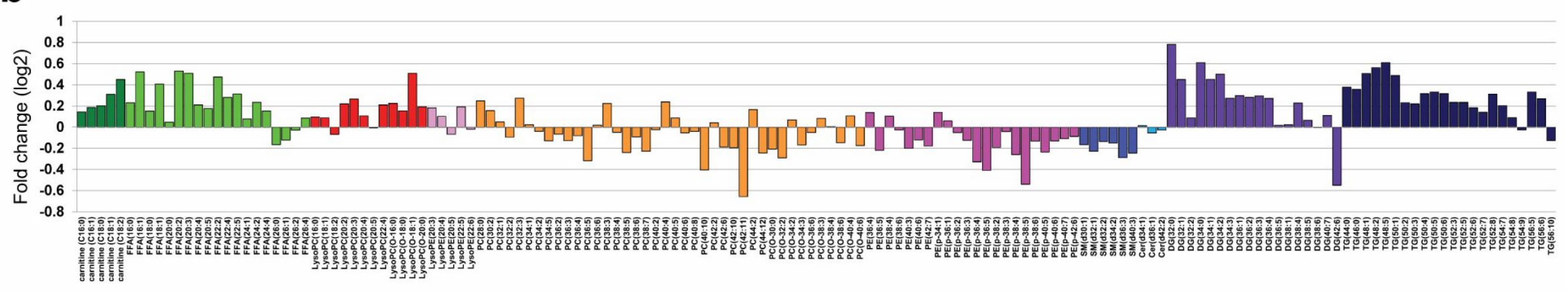

$\square$ Carnitines $\square$ FFAs $\square$ LysoPCs $\square$ LysoPEs $\square$ PCs $\square$ PEs $\square$ SM $\square$ Cers $\square$ DGs $\square$ TGs

Figure S4. ${ }^{1}$ Fold changes in the levels of lipids found in plasma samples from the (a) CIN1 groups and cervical cancer groups and the (b) CIN1 and CIN2/3 groups. FFA: free fatty acid, LysoPC: lysophosphatidylcholine, LysoPE: lysophosphatidylethanolamine, PC: phosphatidylcholine, PE: phosphatidylethanolamine, PI: phosphatidylinositol, SM: sphingomyelin, Cer: ceramide, DG: diglyceride, TG: triglyceride 

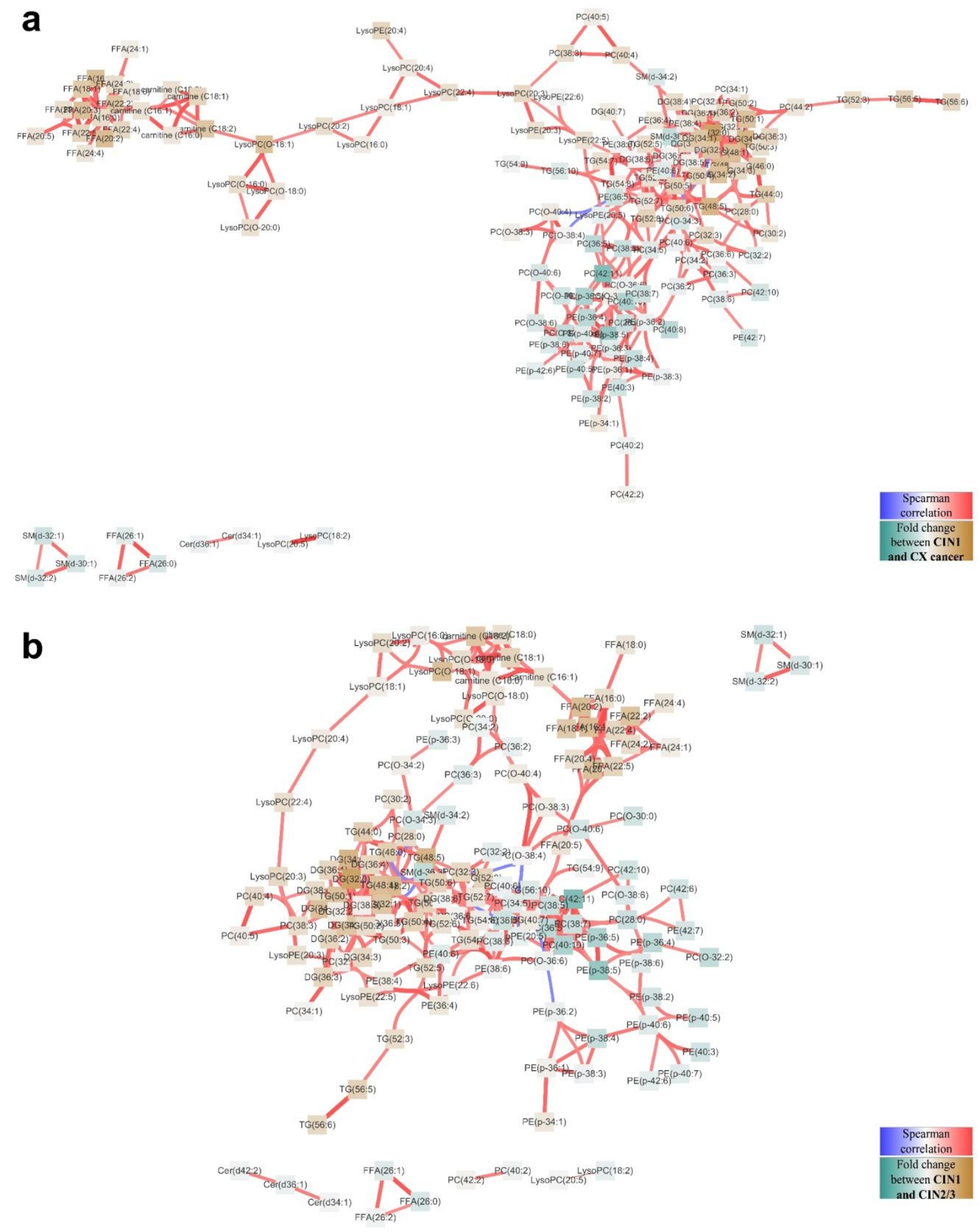

Figure S5. A lipid correlation network was generated between the CIN1 groups and cervical cancer groups (a) and between the CIN1 groups and CIN2/3 groups (b). The correlation network was generated based on the fold change. CIN1: cervical intraepithelial neoplasia 1 , 
CIN2/3: cervical intraepithelial neoplasia 2 or 3, CX cancer: cervical cancer. Edges represent statistically significant Spearman's rank correlation. Red edges indicate Spearman's rank correlation coefficient $>0.6$ and adjusted $\mathrm{P}<0.05$. Blue edges indicate Spearman's rank correlation coefficient $<-0.6$ and adjusted $\mathrm{P}<0.05$.

1. Chung, S.-H.; Franceschi, S.; Lambert, P. F., Estrogen and ER $\alpha$ : Culprits in cervical cancer? Trends in Endocrinology \& Metabolism 2010, 21 (8), 504-511. 